\title{
Routing Hazardous Materials on Time-Dependent Networks using Conditional Value-at-Risk
}

\author{
Iakovos Toumazis Changhyun Kwon* \\ Department of Industrial and Systems Engineering \\ University at Buffalo, SUNY \\ Buffalo, NY, USA
}

\begin{abstract}
We propose a new method for mitigating risk in routing hazardous materials (hazmat), based on the conditional value-at-risk (CVaR) measure on time-dependent vehicular networks. The CVaR models are shown to be flexible and suitable for hazmat transportation that can be solved efficiently. This paper extends the previous research by considering $\mathrm{CVaR}$ for hazmat transportation in the case where accident probabilities and accident consequences are time-dependent. We provide a numerical method to determine an optimal departure time and an optimal route for a given origin-destination pair. The proposed algorithm is tested in a realistic road network in Buffalo, NY, USA and the results are discussed.
\end{abstract}

Keywords: dynamic shortest path; time-dependent network; conditional value-atrisk; hazardous materials transportation

\section{Introduction}

During the last couple of decades, industrial development resulted in the production of enormous quantities of hazardous materials. The U.S. Department of Transportation Pipeline and Hazardous Materials Agency defines hazardous material as a substance or material capable of posing an unreasonable risk to health, safety, or property when transported in commerce (Federal Motor Carrier Safety Administration, 2006). Obviously, these quantities

\footnotetext{
${ }^{*}$ Corresponding author: chkwon@buffalo.edu, +1-716-645-4705
} 
must be transported safely to their final destination. During their transportation, the population, the environment and public structures are exposed to the risk of a potential accident.

In 2007 all commodities shipped were estimated to be 3,344,658,000,000 ton-miles (U.S. Census Bureau, 2007b) with hazardous materials adding up to approximately 323,457,000,000 ton-miles (U.S. Census Bureau, 2007a). Furthermore, hazmat shipments represent about 10 percent of the total commodities shipments ton mileage, and a $5 \%$ increase in hazmat volume each year has been reported (Transportation Research Board, 2005). The average number of miles traveled per hazmat shipment is 96 miles (U.S. Census Bureau, 2007a) whereas the average number of miles traveled per shipment independently of the nature of the load is 619 miles (U.S. Census Bureau, 2007b). These numbers show that hazmat shipments tend to travel shorter distances which along with the operational flexibility of trucks, make them an attractive transportation mode. Despite the fact that only $42.94 \%$ of all hazmat tonnage is transported by truck, a $93.98 \%$ of individual shipments use trucks as a mode of transportation (U.S. Department of Transportation, 1998).

Hazmat accidents are rare events - low-probability incidents with the accident probabilities usually in the range of $10^{-8}$ to $10^{-6}$ per mile traveled (Abkowitz, M. and Cheng, PD, 1988; Harwood et al., 1993) — but with catastrophic consequences (high-consequence incidents) when one does occur. During the year 2011 according to the U.S. Department of Transportation Pipeline and Hazardous Materials Agency, as shown in Table 1, there have been recorded 13,908 hazmat incidents, which resulted in 145 injuries, 10 deaths and damages of total worth $\$ 104,113,342$. Note that the process of hazmat transportation is divided in four phases: loading, in transit, in transit storage and unloading. This study focuses on the transit phase, since the damages caused by incidents during that particular phase had a total cost of $\$ 84,687,976$ along with 70 injuries, among which 12 needed hospitalization. Damages from accidents occurred during all other transportation phases combined had a total cost of $\$ 19,425,366$ and 75 injuries, from which only 11 were hospitalized. More importantly, out of the 10 deaths occurred in 2011, 9 of them resulted from an incident during the transit phase of the transport, while only one occurred during any other phases, namely the loading phase. With more than 800,000 hazardous material shipments performed daily in the U.S. (U.S. Department of Transportation, 1998), the need for risk-averse route decision in the transit phase of hazmat transportation is of great importance.

The static CVaR model (Toumazis et al., 2013) has been recently proposed as an alternative to the existing routing methods. It was shown that CVaR model provides a flexible and riskaverse framework to the decision makers. This paper extends the static model to the dynamic case, and a CVaR minimization model applied in a time-dependent network is proposed. 
Table 1: 2011 Hazmat Summary by Transportation Phase (U.S. Department of Transportation Pipeline and Hazardous Materials Agency)

\begin{tabular}{|c|c|c|c|c|c|}
\hline \multirow{2}{*}{$\begin{array}{c}\text { Transportation } \\
\text { Phase }\end{array}$} & \multirow{2}{*}{ Incidents } & \multicolumn{2}{|c|}{ Injuries } & \multirow{2}{*}{ Fatalities } & \multirow{2}{*}{ Damages } \\
\cline { 3 - 4 } & & Hospitalized & Non-Hospitalized & & \\
\hline Loading & 2,633 & 0 & 22 & 1 & $\$ 793,719$ \\
\hline In Transit & 3,552 & 12 & 58 & 0 & $\$ 84,687,976$ \\
\hline In Transit Storage & 530 & 1 & 6 & 0 & $\$ 17,749,340$ \\
\hline Unloading & 7,193 & 10 & 36 & 10 & $\$ 104,113,342$ \\
\hline Grant Total & 13,908 & 23 & 122 & \\
\hline
\end{tabular}

Specifically, we study the problem in which accident probabilities and accident consequences are time-dependent; that is, the probability of an accident and the resulting consequences depend on the shipment's entrance time in the arc mainly due to traffic conditions.

This manuscript is organized as follows. In Section 2 we provide a brief review of existing hazmat routing models available in the literature and the general concepts of CVaR. In the subsequent section we briefly present the mathematical formulation of the CVaR model applied in a static network. Section 4 develops the formulation of the CVaR model applied in a dynamic network and presents the proposed algorithm. The algorithm is tested in a real vehicular transportation network and the numerical results are illustrated in Section 5 . Finally, Section 6 presents some concluding remarks and future directions of this project.

\section{$2 \quad$ Literature Review}

We begin this section with a brief background on Value-at-Risk (VaR) because CVaR is an extension to VaR. The notion of VaR was initially introduced as a risk measure for overnight risk. Given a confidence level $\alpha \in(0,1)$, the VaR of the portfolio at the confidence level $\alpha$ is given by the smallest number $l$ such that the probability that the loss $L$ exceeds $l$ is at most $(1-\alpha)$. Mathematically, if $L$ is the loss of a portfolio, then $\operatorname{VaR}_{\alpha}(L)$ is the level $\alpha$-quantile (Artzner et al., 1999), i.e.

$$
\operatorname{VaR}_{\alpha}(L)=\inf \{l \in \mathbb{R}: \operatorname{Pr}(L>l) \leq 1-\alpha\}
$$

Despite its wide use and popularity VaR has received criticism because it is not a coherent risk measure (Artzner et al., 1999; Dowd and Blake, 2006) and it might lead to an inaccurate perception of risk (Nocera, 2009; Einhorn, 2008). VaR is also accused for cutting off and ignoring what is taking place in the tail of the distribution as presented by Figure 1. The latter defect of $\mathrm{VaR}$, combined with the fact that hazmat accidents are low-probability 
high-consequence events, will possibly lead VaR model to ignore such road segments from its computations. The VaR model provides flexibility in the risk attitude from risk-indifferent to risk-averse (Kang et al., 2013b; Toumazis et al., 2013). Nevertheless, the risk-indifferent attitude of the VaR model is not favorable in hazmat routing.

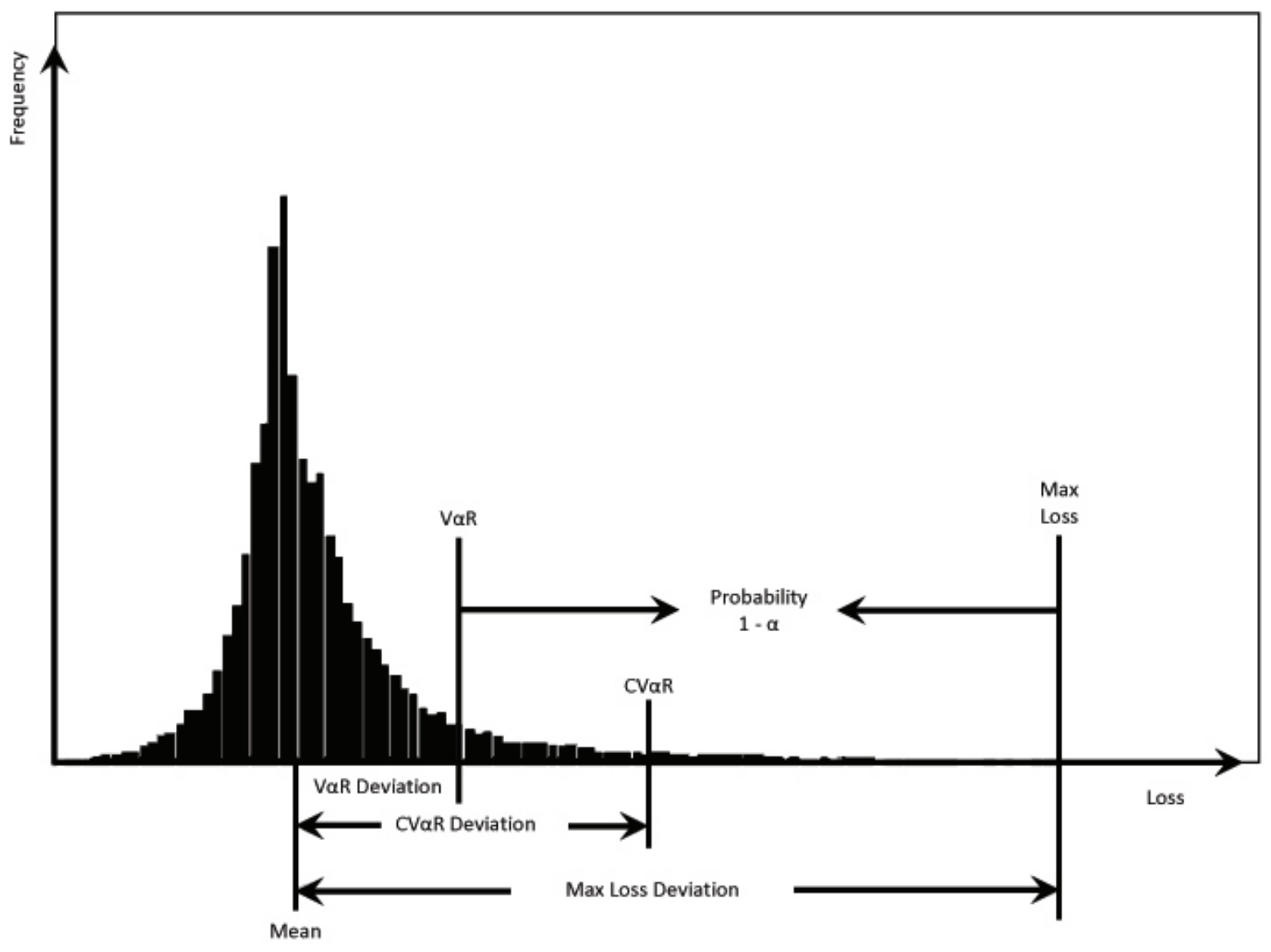

Figure 1: VaR and CVaR Deviations [Source: Sarykalin et al. (2008)]

CVaR is a risk measure that is also broadly used in financial optimization theory. Unlike VaR, CVaR is computationally tractable and coherent risk measure. It mainly focuses on the long tail of the risk distribution to avoid extreme events (see Figure 1), providing a risk-averse tool to the hands of the decision makers when applied in the concepts of hazmat routing. Even though in financial investment problems this may not always lead to the optimal solution; since high risk might result in high profit, that is not the case when applied in hazmat transportation since high risk cannot be traded for high-return. In other words, we consider public safety and therefore, we cannot put people's lives in stake in order to deliver the shipment faster. Therefore, in the case of hazmat transportation a risk-averse approach appears to be more reasonable. 
The concepts of VaR and CVaR applied in hazmat transportation have some significant differences from the respective models utilized in finance. The most distinguished difference between them is that when addressing hazmat transportation problems, the models measure the risk resulted by traversing a particular route in the network. Hence, the investment (that is, the route) and the loss measured (that is the accident consequence) are different quantities, and therefore not comparable. On the contrary, in finance the measurement units of both the investment and the loss are same as, say dollars. In addition, for the models used in hazmat transportation, the risk from traversing each link in a path is non-additive to each other, where in financial models losses of portfolios are additive. It is then obvious, that the models applied in hazmat routing are more complex and require application-specific analysis and computational methods.

The notion of CVaR is also used in facility location problems (Chen et al., 2006) in which the notion is called the mean-excess regret. Furthermore, a similar concept is used in traffic assignment problems (Zhou and Chen, 2008; Wu and Nie, 2011) by the name of mean-excess travel time (METT). METT models consider the travelers' average travel time that exceeds a specified travel time budget in user equilibrium frameworks. Although they are called differently, the notions of mean-excess regret and METT are consistent with CVaR. The METT measure is an alternative to the concept of travel time budget (TTB) (Lo et al., 2006; Shao et al., 2006). Note that the concept of TTB is analogous to VaR, and has received similar criticism as VaR when applied in traffic equilibrium problems.

Chen and Zhou (2010) introduced a new model, called $\alpha$-reliable mean-excess traffic equilibrium (METE) model, which hypothesizes that the commuters are willing to minimize their METT. Their model considers both reliability and unreliability aspects of travel time variability in the commuters' route choice decision process and is formulated as a variational inequality problem. They assumed that the links travel time follow log-normal distributions and derived some properties analytically to formulate the path-finding problem as a chanceconstrained optimization problem. They proposed a route-based solution algorithm, which is in general computationally inefficient.

Many existing hazmat routing methods are available in the literature with the risk imposed by the hazmat shipment transport usually being evaluated by a path evaluation function. A summary of the available models is provided in Table 2. Assume a path $l$ consisting of an ordered set of arcs $(i, j)$, and two attributes - namely, accident probabilities $p_{i j}$ and accident consequences $c_{i j}$-being attached to every arc. The most widely used and easy to implement model, is the Traditional Risk (TR) model, which computes the expected value of the consequences and manipulates it as a risk measure (Sherali et al., 1997). Note that this model assumes that the transport of the shipment ends instantly when an accident occurs 
and the problem is formulated as follows:

$$
\mathbb{E}\left[R^{l}\right]=\sum_{\left(i_{k}, j_{k}\right) \in \mathcal{A}^{l}} \prod_{\left(i_{h}, j_{h}\right) \in \mathcal{A}^{l}, h<k}\left(1-p_{i_{h} j_{h}}\right) p_{i_{k} j_{k}} c_{i_{k} j_{k}}
$$

where $R^{l}$ denotes the accident cost (i.e. risk) associated with path $l, \mathcal{A}^{l}$ denotes the set of links forming path $l$, and $\left(i_{h}, j_{h}\right)$ is the $h^{\text {th }}$ arc of path $l$. However, this formulation is difficult to solve, since it is a nonlinear binary integer problem. Using the fact that the accident probabilities, based on North American data, are on the order of $10^{-6}$ (Abkowitz, M. and Cheng, PD, 1988; Harwood et al., 1993), (2) is approximated by the following simple shortest path problem (Jin and Batta, 1997):

$$
\mathbb{E}\left[R^{l}\right] \approx \sum_{(i, j) \in \mathcal{A}^{l}} p_{i j} c_{i j}
$$

Erkut and Verter (1998) proved that the use of (3) results in a negligible error. Despite the fact that the accident probabilities in North America might be indeed very low, this is not the case in other places of the world or under bad weather conditions (Erkut and Ingolfsson, 2005).

Another model that uses the population exposure concept was introduced by ReVelle et al. (1991). It is called the population exposure (PE) model, and it aims to minimize the total exposure. The Incident Probability (IP) model (Saccomanno and Chan, 1985) assumes constant population densities in the endangered area and minimizes the incident probability. Note that both PE and IP models can be considered as two extreme variations of the TR model.

These models have received criticism due to their assumption of a risk-neutral population. A more risk-averse approach seems more reasonable in the concept of hazmat transportation. Motivated by this, Abkowitz et al. (1992) proposed the Perceived Risk (PR) model, which uses a parameter $q$ to represent risk preference. The introduction of parameter $q$ helps the model avoid arcs that pass through areas with high population density around them, providing a risk-averse framework. However, is difficult to quantify the parameter $q$.

An alternative model that minimizes the expected consequence at the time of the first accident is the Conditional Risk (CR) model (Sivakumar et al., 1993). This model was motivated by the weakness of the TR model to address the problem with multiple hazmat shipments.

Furthermore, Erkut and Ingolfsson (2000) analyzed three additional catastrophe-avoidance modeling methods. The first model, called Maximum Risk (MM) model, focuses on the 
maximum population density exposed to the risk from the transportation of the hazmat shipment. The MM model minimizes the maximum consequence of the proposed path. In other words, it measures how long the tail of the loss distribution extends. The MeanVariance (MV) model is a special case of the PR model, which is popularly used to appraise the trade-offs between return and risk of an investment portfolio. Finally, the Disutility (DU) model employs utility theory to formulate the risk problem in the form of $U(c)=\exp (\lambda c)$, where $\lambda>0$. The DU model provides a risk-averse attitude since the $(i+1)$-st fatality is more costly compared to the $i$-th fatality.

\begin{tabular}{lll}
\hline Model & Risk Measure & Objective \\
\hline TR & Expected Risk & $\min _{l \in \mathcal{P}} \sum_{(i, j) \in \mathcal{A}^{l}} p_{i j} c_{i j}$ \\
PE & Population Exposure & $\min _{l \in \mathcal{P}} \sum_{(i, j) \in \mathcal{A}^{l}} c_{i j}$ \\
IP & Incident Probability & $\min _{l \in \mathcal{P}} \sum_{(i, j) \in \mathcal{A}^{l}} p_{i j}$ \\
PR & Perceived Risk & $\min _{l \in \mathcal{P}} \sum_{(i, j) \in \mathcal{A}^{l}} p_{i j}\left(c_{i j}\right)^{q}$ \\
MM & Maximum Risk & $\min _{l \in \mathcal{P}} \max _{(i, j) \in \mathcal{A}^{l}} c_{i j}$ \\
MV & Mean-Variance & $\min _{l \in \mathcal{P}} \sum_{(i, j) \in \mathcal{A}^{l}}\left(p_{i j} c_{i j}+k p_{i j}\left(c_{i j}\right)^{2}\right)$ \\
DU & Disutility & $\min _{l \in \mathcal{P}} \sum_{(i, j) \in \mathcal{A}^{l}} p_{i j}\left(\exp \left(k c_{i j}-1\right)\right)$ \\
CR & Conditional Probability & $\min _{l \in \mathcal{P}}\left(\sum_{(i, j) \in \mathcal{A}^{l}} p_{i j} c_{i j} / \sum_{(i, j) \in \mathcal{A}^{l}} p_{i j}\right)$ \\
\hline
\end{tabular}

Table 2: Classic Path Risk Evaluation Models (Erkut and Ingolfsson, 2005)

Even though an enormous number of publications addresses the shortest path problem in networks, the majority of these publications consider static networks that have fixed arc costs. However, in recent years the interest in shortest path problems has been renewed, resulting in a number of publications dealing with shortest path problems in time-dependent networks (Miller-Hooks and Mahmassani, 2000; Ahuja et al., 2002; Chabini and Ganugapati, 2002; Ahuja et al., 2003). The most notable difference between the two versions of shortest path problems is that in the dynamic case the arc costs and the arc travel times depend on the time of entrance in the arc. On the other hand, in the static case arc costs and travel times are fixed constants. Dynamic shortest path problems have been a very powerful tool used for Intelligent Transportation Systems (ITS), real-time dynamic management and route guidance models. 
The first publication studying shortest path algorithms (Cooke and Halsey, 1966) proposed an extension of Bellman's principle of optimality that was computing the shortest route between every node in the network to the destination node for different time-steps. However, no numerical results exist to study the efficiency of the proposed method. Another approach with the same complexity as Cooke and Halsey (1966) when trying to compute the shortest path from every node to the destination, was proposed by Dreyfus (1969). Dreyfus's approach computes the shortest path for a single time step between a unique origin-destination pair. In order for this algorithm to be able to detect the shortest path, the First-In-First-Out (FIFO) condition must hold for every arc.

An additional algorithm calculating the time-dependent shortest paths from all nodes in the network to the destination node is proposed by Ziliaskopoulos and Mahmassani (1993). The algorithm is based on Bellman's principle of optimality and the paths are calculated while the algorithm operates backwards in a label correcting way. The big advantage of this algorithm is that it can be applied in networks in which arc costs are not necessarily travel times. Following the work of Ziliaskopoulos and Mahmassani (1993) many additional studies on label correcting algorithm were presented. For example, Miller-Hooks and Mahmassani (1998, 2000) and Nie and Wu (2009) apply the label correcting algorithm with some modifications in stochastic, time-varying networks, where Andersen and Skriver (2000) uses the label correcting algorithm to solve the bicriterion shortest path probelm.

Also, a large number of publications exists addressing hazmat transportation problems. The majority of these manuscripts addresses vehicle routing and scheduling problems (Abkowitz, M. and Cheng, PD, 1988; Abkowitz et al., 1992; Sherali et al., 1997; Miller-Hooks and Mahmassani, 1998; Zografos et al., 2002; Chang et al., 2005; Zografos and Androutsopoulos, 2008; Androutsopoulos and Zografos, 2010; Xie et al., 2012; Toumazis and Kwon, 2013; Kwon et al., 2013), while others address the problem of network design (Kara and Verter, 2004; Erkut and Alp, 2007; Erkut and Gzara, 2008; Verter and Kara, 2008; Bianco et al., 2009). For a comprehensive literature review, see Erkut et al. (2007).

\section{CVaR in Static Networks}

CVaR applied in the concept of hazardous materials transportation has been recently proposed (Toumazis et al., 2013; Toumazis and Kwon, 2013). In this section we introduce the CVaR concept in time-invariant networks and provide some key characteristics of CVaR with formal proofs. Assume a directed and weighted network $G=(\mathcal{N}, \mathcal{A})$, where $\mathcal{N}$ is the set of nodes and $\mathcal{A}$ the set of directed arcs. Let $\mathcal{P}$ denote the set of all alternative paths in the network. For a path $l \in \mathcal{P}$ at the confidence level $\alpha$, the CVaR for general distributions is defined as 
(Rockafellar and Uryasev, 2002; Sarykalin et al., 2008):

$$
\mathrm{CVaR}_{\alpha}^{l}=\lambda_{\alpha}^{l} \operatorname{VaR}_{\alpha}^{l}+\left(1-\lambda_{\alpha}^{l}\right) \mathbb{E}\left[R^{l} \mid R^{l}>\operatorname{VaR}_{\alpha}^{l}\right]
$$

Note that $\lambda_{\alpha}^{l}=\left(\operatorname{Pr}\left[R^{l} \leq \operatorname{VaR}_{\alpha}^{l}\right]-\alpha\right) /(1-\alpha)$, and $\operatorname{VaR}$ in hazmat transportation is defined as $\operatorname{VaR}_{\alpha}^{l}=\min \left\{\beta: \operatorname{Pr}\left(R^{l}>\beta\right) \leq 1-\alpha\right\}$; the minimum level $\beta$ such that the probability of the risk $R^{l}$, when transporting path $l$, being more than $\beta$ is less than or equal to $1-\alpha$.

$\mathrm{CVaR}_{\alpha}^{l}$ given in the form (4) cannot be used in an optimization problem because of the conditioning in the expectation. However, Rockafellar and Uryasev (2000) showed that the CVaR minimization problem is equivalent to the following function (Rockafellar and Uryasev, 2000; Pflug, 2000)

$$
\Phi_{\alpha}^{l}(r)=r+\frac{1}{1-\alpha} \mathbb{E}\left[R^{l}-r\right]^{+} \approx r+\frac{1}{1-\alpha} \sum_{(i, j) \in \mathcal{A}^{l}} p_{i j}\left[c_{i j}-r\right]^{+}
$$

when minimized by choosing a path $l \in \mathcal{P}$ at the confidence level $\alpha$. Note that $[x]^{+}=$ $\max (x, 0), p_{i j}$ is the accident probability on arc $(i, j)$, and $c_{i j}$ the accident consequence on $\operatorname{arc}(i, j)$. That is,

$$
\min _{l \in \mathcal{P}} \mathrm{CVaR}_{\alpha}^{l}=\min _{l \in \mathcal{P}, r \in \mathbb{R}^{+}} \Phi_{\alpha}^{l}(r)
$$

Also, the parameter $r$ was shown to be equal to the VaR value of the proposed path for the same confidence level (Rockafellar and Uryasev, 2000). The CVaR minimization problem can be written as

$$
\min _{r \in \mathbb{R}^{+}}\left(r+\frac{1}{1-\alpha} z_{\alpha}(r)\right)
$$

where $z_{\alpha}(r) \equiv \min _{x \in \Omega} \sum_{(i, j) \in \mathcal{A}} p_{i j}\left[c_{i j}-r\right]^{+} x_{i j}$, and

$$
\Omega \equiv\left\{x: \sum_{(i, j) \in \mathcal{A}} x_{i j}-\sum_{(j, i) \in \mathcal{A}} x_{j i}=b_{i} \quad \forall i \in \mathcal{N}, \text { and } x_{i j} \in\{0,1\} \quad \forall(i, j) \in \mathcal{A}\right\}
$$

The parameter $b_{i}$ takes the following values:

$$
b_{i}= \begin{cases}1 & , \text { if node } i \text { is the source } \\ -1 & , \text { if node } i \text { is the sink } \\ 0 & , \text { otherwise }\end{cases}
$$

Following the mathematical analysis of (5), we end up with the following minimization 
problem:

$$
\mathrm{CVaR}_{\alpha}^{*}=\min _{r=0, c_{(1)}, \ldots, c_{(\bar{m})}}\left[r+\frac{1}{1-\alpha} \min _{x \in \Omega} \sum_{(i, j) \in \mathcal{A}} p_{i j}\left[c_{i j}-r\right]^{+} x_{i j}\right]
$$

which may be solved by a finite number of shortest-path problems (Toumazis et al., 2013). Note that $c_{(k)}$ is the $k$-th smallest value in the set $\left\{c_{i j}:(i, j) \in \mathcal{A}\right\}$, where we define $c_{(0)}=0$ and $\bar{m}=|\mathcal{A}|$; the cardinality of set $\mathcal{A}$. CVaR model offers a flexible tool for decision makers whose risk attitude ranges from risk-neutral to risk-averse. That is, the model proposes different optimal routes for different confidence levels.

Next, we provide the properties that CVaR model possesses.

Lemma 1. For any $\alpha \in(0,1)$ and path $l \in \mathcal{P}$, if $\operatorname{VaR}_{\alpha}^{l}=0$, then $\mathrm{CVaR}_{\alpha}^{l}=\sum_{(i, j) \in \mathcal{A}^{l}} p_{i j} c_{i j}$.

Proof. The proof of Lemma 1 is provided in Appendix A. All other omitted proofs can be found in Appendix A as well.

Lemma 2. For any $\alpha \in(0,1)$ and path $l \in \mathcal{P}$, if $\operatorname{VaR}_{\alpha}^{l}=\max _{(i, j) \in \mathcal{A}^{l}} c_{i j}$, then $\mathrm{CVaR}_{\alpha}^{l}=$ $\mathrm{VaR}_{\alpha}^{l}=\max _{(i, j) \in \mathcal{A}^{l}} c_{i j}$.

Lemma 1 states that for any given path $l \in \mathcal{P}$ and any confidence level value, the CVaR model for path $l$, when the respective $\mathrm{VaR}$ model is not effective; i.e. $\mathrm{VaR}_{\alpha}^{l}=0$, is equivalent to the Traditional Risk (TR) model. Similarly, Lemma 2 states that when VaR model is equivalent to the Maximum Risk (MM) model; i.e. VaR value equals the maximum consequence among the $\operatorname{arcs}(i, j)$ composing path $l$, the CVaR model is equivalent to the Maximum Risk (MM) model.

Theorem 1. There exists a scalar $\alpha_{\min }$, such that $l_{\mathrm{CVaR}}^{*}=l_{\mathrm{TR}}^{*}, \forall \alpha \in\left(0, \alpha_{\min }\right]$, where $l_{\mathrm{CVaR}}^{*}$ and $l_{\mathrm{TR}}^{*}$ are the optimal paths determined by the CVaR model and the Traditional Risk model respectively. In particular,

$$
\alpha_{\min }=\min _{l \in \mathcal{P}}\left(1-\sum_{(i, j) \in \mathcal{A}^{l}} p_{i j}\right)
$$

Theorem 2. There exists a scalar $\alpha_{\max }$, such that $l_{\mathrm{CVaR}}^{*}=l_{\mathrm{MM}}^{*}, \forall \alpha \in\left(\alpha_{\max }, 1\right)$, where $l_{\mathrm{CVaR}}^{*}$ and $l_{\mathrm{MM}}^{*}$ are the optimal paths determined by the CVaR model and the Maximum Risk model respectively. In particular,

$$
\alpha_{\max }=\max _{l \in \mathcal{P}} p_{\max }^{l}
$$

where $p_{\max }^{l}=\operatorname{Pr}\left[R^{l}=\max _{(i, j) \in \mathcal{A}^{l}} c_{i j}\right]$ for all $l \in \mathcal{P}$, which is the accident probability of the link with the greatest accident consequence in a path $l$. 
In other words, Theorem 1 says that the proposed path obtained by the CVaR model is the same as the path that the TR model proposes when the confidence level value is less

than $\alpha_{\text {min }}=\min _{l \in \mathcal{P}}\left(1-\sum_{(i, j) \in \mathcal{A}^{l}} p_{i j}\right)$. On the other hand, Theorem 2 states that when the confidence level value is greater than $\alpha_{\max }=\max _{l \in \mathcal{P}} p_{\max }^{l}$ then the proposed path from the CVaR model is the same as the one obtained from the MM model. Therefore, we observe that the CVaR model offers a flexible tool for decision makers whose risk attitudes are risk-neutral (TR model) to risk-averse (MM model).

\section{CVaR Minimization Model for a Dynamic Network}

In this part of the manuscript, we provide the mathematical formulation of the model when applied in a time-dependent network. In Section 4.1 we describe the methodology implemented to express time in a discrete form, followed by the definition of CVaR in a time-dependent network in Section 4.2. Section 4.3 presents the algorithm used to solve the dynamic shortest path subproblem, and in Section 4.4 we give the proposed algorithm for the main CVaR minimization problem. Furthermore, some of the properties of the model supporting the suitability of the algorithm are presented in Section 4.4.

\subsection{Discrete Network}

Assume a directed, weighted, discrete FIFO dynamic network $G=(\mathcal{N}, \mathcal{A})$, where $\mathcal{N}$ is the set of nodes and $\mathcal{A}$ the set of directed arcs. Let

$$
d_{i j}(t, r)=p_{i j}(t)\left[c_{i j}(t)-r\right]^{+}
$$

be the non-negative time-dependent arc cost, i.e. risk, from traversing arc $(i, j)$ at time $t$. Note that $d_{i j}(t, r)$ is the "travel cost" (risk) by traversing arc $(i, j)$ when the entrance time is $t$ for a given scalar $r$ and it is a real-valued function, which is defined for every $t \in S$, where

$$
S=\left\{t_{0}, t_{0}+\delta, t_{0}+2 \delta, \ldots, t_{0}+M \delta\right\}
$$

In addition, let $\tau_{i j}(t)$ denote the time needed to traverse arc $(i, j)$ when the entrance time in the arc is at time $t$. The earliest possible departure time, from any node of the network is denoted as $t_{0}$. Constants $\delta$ and $M$ are both user defined and represent a small time interval during which some meaningful change in the traffic conditions may occur, and a large integer value such that the time interval from $t_{0}$ to $t_{0}+M \delta$ covers the desirable time period understudy respectively. 
When the entrance time to a certain arc is not in the set $S$, then the travel cost is computed by considering the closest time index that is earlier than the actual entrance time. That is, for an entrance time $t$ to an arc $(i, j)$, we take $t^{\prime} \in S$ that is the closest element to $t$ such that $t^{\prime} \leq t$, and compute

$$
d_{i j}(t, r)=p_{i j}\left(t^{\prime}\right)\left[c_{i j}\left(t^{\prime}\right)-r\right]^{+}
$$

The same method is used to compute $\tau_{i j}(t)$. Therefore both the travel cost and time take discrete values.

We use the discrete time index set because the available data for all existing road networks are already discrete. In addition, the use of the discrete time index set brings computational advantages. As we will see in the following subsections, it is computationally efficient to obtain an optimal departure time and an optimal path for the CVaR minimization problem.

\subsection{CVaR Defined for a Time-Dependent Network}

For a path $l$, the CVaR measure becomes as follows for any departure time $t$ from the origin:

$$
\operatorname{CVaR}_{\alpha}^{l}(t)=\min _{r \in \mathbb{R}^{+}}\left(r+\frac{1}{1-\alpha} \sum_{(i, j) \in \mathcal{A}^{l}} d_{i j}\left(\theta_{i j}^{l}(t), r\right)\right)
$$

where $\theta_{i j}^{l}(t)$ is the time moment at which a truck enters the arc $(i, j)$ when it departed from the origin at time $t$. We can express:

$$
\begin{aligned}
& \theta_{i_{1} j_{1}}^{l}(t)=t \\
& \theta_{i_{2} j_{2}}^{l}(t)=t+\tau_{i_{1} j_{1}}\left(\theta_{i_{1} j_{1}}^{l}(t)\right)=t+\tau_{i_{1} j_{1}}(t) \\
& \theta_{i_{3} j_{3}}^{l}(t)=\theta_{i_{2} j_{2}}^{l}(t)+\tau_{i_{2} j_{2}}\left(\theta_{i_{2} j_{2}}^{l}(t)\right)=t+\tau_{i_{1} j_{1}}(t)+\tau_{i_{2} j_{2}}\left(t+\tau_{i_{1} j_{1}}(t)\right)
\end{aligned}
$$

where $\left(i_{k}, j_{k}\right)$ represents the $k$-th arc in path $l$.

Our problem in a time-dependent network is to seek a path and a departure time that minimizes the CVaR measure of hazmat transportation risk:

$$
\begin{aligned}
\min _{l \in \mathcal{P}, t \in S} \operatorname{CVaR}_{\alpha}^{l}(t) & =\min _{l \in \mathcal{P}, t \in S} \min _{r \in \mathbb{R}^{+}}\left(r+\frac{1}{1-\alpha} \sum_{(i, j) \in \mathcal{A}^{l}} d_{i j}\left(\theta_{i j}^{l}(t), r\right)\right) \\
& =\min _{r \in \mathbb{R}^{+}}\left(r+\frac{1}{1-\alpha} z_{\alpha}(t, r)\right)
\end{aligned}
$$


where

$$
z_{\alpha}(t, r)=\min _{l \in \mathcal{P}, t \in S} \sum_{(i, j) \in \mathcal{A}^{l}} d_{i j}\left(\theta_{i j}^{l}(t), r\right)
$$

Note that the set $\Omega$ and the parameter $b_{i}$ take the same values as in the static case described in previous section in (6) and (7) respectively.

Subproblem (15) is a shortest path problem in a time-dependent network with arc cost being equal to $d_{i j}\left(\theta_{i j}^{l}(t), r\right)$, and the arc travel time defined by $\tau_{i j}(t)$. Therefore, for each value of $r$ we can evaluate $z_{\alpha}(t, r)$ by solving a dynamic shortest path problem.

\subsection{Solving the Dynamic Shortest-Path Sub-Problems}

As stated at the beginning of this section, $G=(\mathcal{N}, \mathcal{A})$ is a discrete FIFO dynamic network. Therefore, the FIFO condition must be satisfied for each arc and every time step of the network. Hence, we have the following set of equations that must hold for each arc and every time step:

$$
\forall(i, j, t), \quad t+\tau_{i j}(t) \leq(t+1)+\tau_{i j}(t+1)
$$

In other words, what the set of inequalities in (16) is saying is that no overpassing is allowed in the network.

In this paper, we use a back-labeling algorithm proposed by Ziliaskopoulos and Mahmassani $(1993)^{1}$ that is based on Bellman's principle of optimality. It calculates for every time step $t \in S$ the time-dependent shortest paths from every node $i$ in the network to the destination node $N$. The most noteworthy difference of this approach is that it can deal with networks with the arc cost not necessarily being the travel times. This advantage motivated us to use the particular algorithm, with the arc cost being equal to the risk exposed by traversing each arc in the network. Next we present a slightly modified version of the algorithm that effectively and efficiently solves the time-dependent inner shortest path of the CVaR minimization problem in hazmat transportation.

In order for the algorithm to work some assumptions had to be made (Ziliaskopoulos and Mahmassani, 1993). First of all, we assume that $\tau_{i j}(t)=\tau_{i j}\left(t_{0}+M \delta\right)$ is constant for all $t>t_{0}+M \delta$. In other words, we assume that the traffic conditions in the understudy transportation network after the peak hour are stable. In addition, it is assumed that, $\tau_{i j}(t)=\tau_{i j}\left(t_{0}+k \delta\right) \forall t \in\left(t_{0}+k \delta, t_{0}+(k+1) \delta\right)$. That is, the travel time required to traverse arc $(i, j)$ remains the same for any departure time within a time step. Note at this point that the first two assumptions are not restrictive, since as stated before, constants $\delta$ and $M$ are

\footnotetext{
${ }^{1}$ A similar version of the algorithm was also used by Miller-Hooks and Mahmassani (1998, 2000); Nie and $\mathrm{Wu}(2009)$
} 
user defined and therefore, can always change. Hence, the user may increase constant $M$ in such a way, that the time interval understudy extends to include periods with variable risk exposition on some of the arcs. Also, constant $\delta$ can be set to a very small value such that the traffic conditions remain unchanged within a time step.

At each computational step of the algorithm the total risk of the current shortest path from node $i$ to node $N$ at time $t$ is denoted by $\psi(t)$. The $M$-vector label $\Psi_{i}=\left[\psi_{i}\left(t_{0}\right), \psi_{i}\left(t_{0}+\right.\right.$ $\left.\delta), \psi_{i}\left(t_{0}+2 \delta\right), \ldots, \psi_{i}\left(t_{0}+M \delta\right)\right]$, contains all the labels for every time step $t$ for node $i$. Using the ordered set of nodes $P_{i}=\left\{i=n_{1}, n_{2}, \ldots, n_{m}=N\right\}$, we can identify any label $\psi_{i}(t)$ from node $i$ to the destination node $N$. We use the following formula to define $\psi_{i}(t)$ (Miller-Hooks and Mahmassani, 1998, 2000; Nie and Wu, 2009):

$$
\psi_{i}(t)= \begin{cases}\min _{j \neq i}\left\{\psi_{j}\left(t+\tau_{i j}(t)\right)+d_{i j}(t)\right\} & , \text { for } i=1,2, \ldots, N-1 \text { and } \forall t \in S \\ 0 & , \text { for } i=N \text { and } \forall t \in S\end{cases}
$$

The proposed algorithm segments the time period understudy into small discrete time intervals $\delta$. It begins from the destination node $N$, and calculates the optimal routes operating in a backward label-correcting way. In order to avoid scanning every node of the network in every iteration, it uses a scan eligible (SE) list, which contains all the nodes of the network that might have at least one label improved. Note that, for the creation, insertion, and deletion of a node in the SE list we follow specific rules (Dial et al., 1979). Because the algorithm operates in a label-correcting fashion the label vectors are upper bounds to the shortest paths until the algorithm concludes to the optimal solution.

The modified algorithm applied to the dynamic version of the CVaR model proceeds with the following steps for every value of $r$ :

Step 1: Create the SE list and place into it the destination node $N$. Let $\Psi_{N}=(0,0, \ldots, 0)$ and $\Psi_{i}=(\infty, \infty, \ldots, \infty)$ for $i=1,2, \ldots, N-1$

Step 2: Choose the first node $i$ in the SE list, name it 'Current Node', and remove it from the list. If the SE list is empty, go to Step 4. Otherwise, scan the current node $i$ according to the following equation

$$
\psi_{j}(t)=\min \left\{\psi_{j}(t), d_{j i}(t)+\psi_{i}\left(t+\tau_{j i}(t)\right)\right\}, j \in \Gamma^{-1}\{i\} \text { for all } t \in S
$$

Specifically, for every time step $t \in S$, if $\psi_{j}(t)$ is greater than $d_{j i}(t)+\psi_{i}\left(t+\tau_{j i}(t)\right)$ replace $\psi_{j}(t)$ in the label vector $\Psi_{j}$ at position i with the new value. If any of the $\mathrm{M}$ labels of node $\mathrm{j}$ has been improved, insert node $\mathrm{j}$ in the SE list.

Step 3: Repeat Step 2. 
Step 4: Stop; the vector $\Psi_{i} \forall i \in \mathcal{A}$ contain the risk of the proposed routes for each time step $t \in S$.

The following theorem holds (Ziliaskopoulos and Mahmassani, 1993): At the completion of the algorithm, every element of the vector $\Psi_{N}$ is either an infinite number, indicating that there is no path from this node to the destination node at the corresponding time step, or a finite number that represents the risk exposed from traversing the shortest path from this node and the time step to the destination node.

The efficiency of the algorithm depends on the total number of scanned nodes before the completion of the algorithm. Obviously, the total number of nodes in the network, $|\mathcal{N}|$, is the lower bound of the total number of nodes scanned, with the upper bound being equal to $|\mathcal{N}|^{2} M$ (Ziliaskopoulos and Mahmassani, 1993). In the case where the maximum indegree of a node is $|\mathcal{N}|-1$, the worst-case running time complexity of the algorithm is $O\left(M^{2}|\mathcal{N}|^{3}\right)$ (Ziliaskopoulos and Mahmassani, 1993).

\subsection{Solving the Main Problem}

Note that the above algorithm provides us with the solution to the dynamic shortest path problem $z_{\alpha}(t, r)$ in (15) for any given value of $r$. For the computation of the optimal $r$ value, a similar argument as in the static case is used. Since we use the discrete time index set $S$, the accident consequence will have a value from the following discrete set:

$$
\widehat{C}=\{0\} \cup\left\{c_{i j}(t):(i, j) \in \mathcal{A}, t \in S\right\}
$$

Let us sort the elements of $\widehat{C}$ in an ascending order, so that

$$
\widehat{C}=\left\{c_{(k)}: k=0,1,2, \ldots,|\mathcal{A}| \times|S|\right\}
$$

where $c_{(0)}=0$, and $c_{(k)}<c_{(k+1)}$ for all $k$. Then we obtain the following result:

Lemma 3. For any path $l \in \mathcal{P}$ and for any departure time $t \in S$, a solution $r^{*}$ to the following problem

$$
\operatorname{CVaR}_{\alpha}^{l}(t)=\min _{r \in \mathbb{R}^{+}}\left(r+\frac{1}{1-\alpha} \sum_{(i, j) \in \mathcal{A}^{l}} d_{i j}\left(\theta_{i j}^{l}(t), r\right)\right)
$$

has a value from the set $\widehat{C}$.

Using Lemma 3, the following main result is immediate: 
Theorem 3. A solution $r^{*}$ for the CVaR minimization problem in a time-dependent network:

$$
\min _{l \in \mathcal{P}, t \in S} \operatorname{CVaR}_{\alpha}^{l}(t)=\min _{l \in \mathcal{P}, t \in S} \min _{r \in \mathbb{R}^{+}}\left(r+\frac{1}{1-\alpha} \sum_{(i, j) \in \mathcal{A}^{l}} d_{i j}\left(\theta_{i j}^{l}(t), r\right)\right)
$$

has a value from the set $\widehat{C}$.

Therefore, by examining all elements in the set $\widehat{C}$ we can obtain an optimal solution to the CVaR minimization problem in a time-dependent network. In particular, we propose the following algorithm:

Step 0. Order the set $\widehat{C}$ in an ascending order, and set $w^{\sharp} \leftarrow \infty$. Set $k=0$.

Step 1. Let $r_{k}=c_{(k)}$, and solve the dynamic shortest-path sub-problem $z_{\alpha}\left(t, r_{k}\right)$ in (15). Set $w^{\sharp} \leftarrow \min \left\{w^{\sharp}, r_{k}+\frac{1}{1-\alpha} z_{\alpha}\left(t, r_{k}\right)\right\}$.

Step 2. Terminate the algorithm and declare the current $w^{\sharp}$ as the optimal CVaR and the corresponding departure time and path as solutions, if either of the following conditions holds:

1. All elements of the set $\widehat{C}$ have been examined.

2. $c_{(k+1)} \geq w^{\sharp}$.

Otherwise, set $k \leftarrow k+1$ and go to Step 1 .

The theoretical worst-case complexity of the above algorithm is the product of the worst-case complexity of the algorithm presented in Section 4.3 times the number of elements in the set $\widehat{C}$; that is, $O\left(M^{2}|\mathcal{N}|^{3}|\widehat{C}|\right)$.

Note that we use $w^{\sharp}$ to keep the incumbent minimal CVaR found so far. If the second termination condition $c_{(k+1)} \geq w^{\sharp}$ holds, the current $w^{\sharp}$ will not improve, because $r_{(k+1)}$ is already no less than $w^{\sharp}$ and $z_{\alpha}$ is always nonnegative. By employing this second condition, we can reduce the computational efforts. Obviously, this algorithm will terminate after solving at most $|S| \times|\mathcal{A}|$ dynamic shortest-path sub-problems.

\section{Case Study}

In the upcoming sections the findings of a case study are presented. First, we provide the characteristics of the understudy network along with the methods used to establish the input data in Section 5.1. In Section 5.2 we present the results obtained under two different data sets: ignoring the network's infrastructure and considering it. Then, sensitivity analyses on the models' parameters are provided in Section 5.3. 


\subsection{Buffalo Network and Data Analysis}

To illustrate the findings of this research a case study was developed in a part of the transportation network of Buffalo, NY. The network consists of 90 nodes, 149 arcs, a unique origin-destination pair, and a single hazmat shipment. The time period understudy was from 8 am to 11 am and the reason for our choice was to capture the morning road congestion. We divided the time period of interest into 5-minute time steps using linear approximation to the raw data. That means that $\delta=5$ minutes and in order to cover the 3 hour time horizon, the constant $M=36$. Note that the approximation utilized is described in detail in Section 5.1.2. The colored background in Figure 2 represents the population densities in the Buffalo area in 2010. As shown in the legend, the darker the color is the higher the population density in the area. In addition, Figure 2 demonstrates the traffic conditions based on the average traffic volume in the 3 hour span understudy.

The representation of the CVaR problem for a dynamic network is more complicated than the respective problem applied in a static network, since in this case we have to specify for each arc the corresponding accident probabilities and consequences for all time steps ( $M$ total steps).

Next, we describe the methods used to compute these two parameters along with the method used for the computation of the arcs travel times.

\subsubsection{Computation of Accident Probabilities $p_{i j}$}

Assume that the number of hazmat accidents at the shipment's entrance time, $t$, in arc $(i, j)$, $\mathcal{E}_{i j}^{t}$, follows Poisson distribution with parameter:

$$
\begin{aligned}
\gamma_{i j}(t) & =(\text { hazmat accident rate per mile } / \text { vehicle }) \times(\text { arc length }) \times(\text { traffic volume at time } \mathrm{t}) \\
& =\left(3.19922 \times 10^{-7}\right) l_{i j} V_{i j}(t)
\end{aligned}
$$

Therefore, we can write

$$
\mathcal{E}_{i j}^{t} \sim \operatorname{Poisson}\left(\gamma_{i j}(t)\right)
$$

Note here that the hazmat accident rate per mile/vehicle $=3.19922 \times 10^{-7}$ (Federal Motor Carrier Safety Administration, 2001), $l_{i j}$ is the length of arc $(i, j)$, and $V_{i j}(t)$ is the traffic volume at time $t$ on $\operatorname{arc}(i, j)$. The latter assumption was made after considering the nature of hazmat accidents. It is known that the Poisson distribution is the most appropriate distribution for rare events, and therefore a proper choice for the number of hazmat accidents. In addition, if we examine closely the selection of the parameter $\gamma_{i j}(t)$, we will see that it is basically the expected number of accidents for each link at every time step $t$. In other 


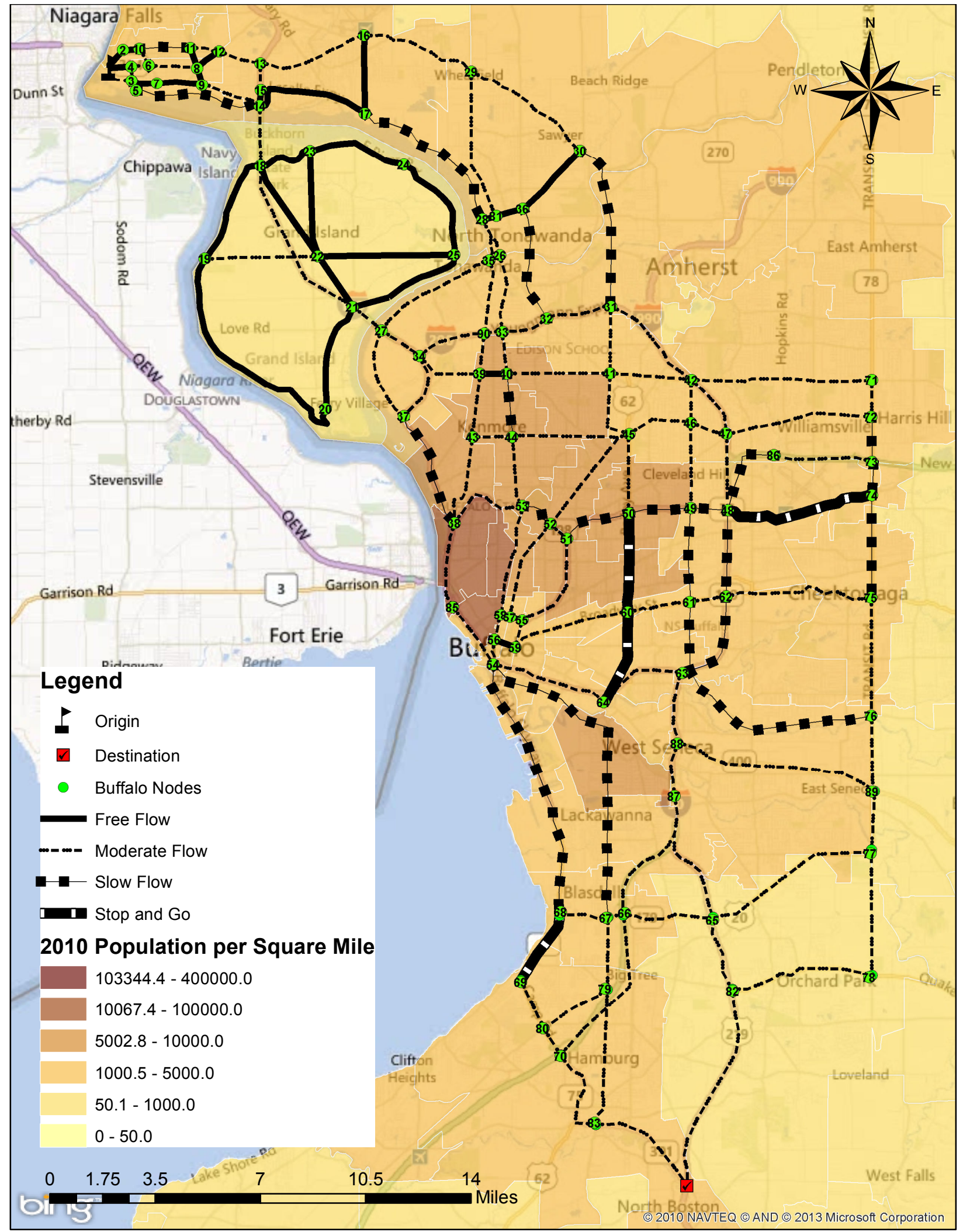

Figure 2: Case Study Network 
words, the parameter $\gamma_{i j}(t)=E\left(\mathcal{E}_{i j}^{t}\right)$. Adopting the latter assumption allows us to use the Poisson probability generating function for the computation of the time-dependent accident probabilities, for every arc and every time step. The following formula was used:

$$
p_{i j}(t)=1-\operatorname{Pr}\{\text { No accident occurs. }\}=1-\frac{\gamma_{i j}(t)^{\epsilon}}{\epsilon !} e^{-\gamma_{i j}(t)}
$$

where $\epsilon=0$. Expression (21) shows that the probability of one accident or more is equal to 1 minus the probability of no accident on $\operatorname{arc}(i, j)$.

\subsubsection{Traffic Volume Data $V_{i j}(t)$}

The traffic volume raw data we collected from the website of the New York State Department of Transportation were initially in 60-minute intervals. However, we converted these data to match our 5 minute time steps by increasing or decreasing the traffic volume gradually, segmenting the difference of two consecutive hourly data values to 12 discretized 5-minute time slots. For example, assume that our traffic volume data for two consecutive hours are 140 and 200. To transform these hourly data into 5-minute data, we proceed as follows: First we take the difference of the two values: $200-140=60$. If the result is positive, then we will have an increase in the traffic volume for all twelve 5 -minute time periods. If negative, then we will have a decrease in the 5 -minute traffic volume periods. We divide that number by the number of 5 -minute periods within the hour, i.e. $60 / 12=5$, rounding the result to the next integer. That gives us the amount of increase (or decrease) from each 5-minute time step to the next one. Finally, in our example we will have 140, 145, $150 \ldots, 200$ as the 12 discretized 5-minute periods traffic volume. When all 12 time periods are computed we repeat the same procedure for the next hour. Even though the traffic volume changes are not linear within an hour, it was assumed so for simplicity purposes.

\subsubsection{Travel Times Calculation}

Furthermore, based on the 5 minute traffic volume data we had to compute the congested travel times for each arc in the network. In order to do that, the Bureau of public Roads (BPR) function was used (Branston, 1976):

$$
\tau_{i j}(t)=\tilde{t}\left(1+0.15\left(\frac{V_{i j}(t)}{\tilde{c}_{i j}}\right)^{4}\right)
$$

where $\tilde{t}$ is the free flow travel time on $\operatorname{arc}(i, j), V_{i j}(t)$ is the traffic volume on arc $(i, j)$ at time $t$, and $\tilde{c}_{i j}$ is the capacity of arc $(i, j)$. Note that the free flow travel time was computed by the formula $\tilde{t}=l_{i j} /$ (speed limit), with $l_{i j}$ representing the length of arc $(i, j)$. The data 
for the speed limits and arc capacities were obtained from the New York State Department of Transportation website.

\subsubsection{Computation of Accident Consequences}

For the computation of the time-dependent accident consequences we estimated the weighted sum of the population density in the hazmat impact zone and the traffic volume in the same area at the time of the accident. We considered a circle of radius $\lambda$ that is equal to the hazmat spread radius as shown in Figure 3, which is commonly used in the literature (Erkut and Verter, 1998; Erkut et al., 2007). For different types of hazmat, different values of $\lambda$ radius may be used. However, because this paper addresses the problem of transporting a single shipment, it is assumed that the accident impact zone is independent from the shipment's hazmat type. In addition, the population density of every area changes during the day: people are going to work at a different place, children go to school etc. Nonetheless, we did not consider such time-variant population density factors in this paper, as estimating such population shifts is out of scope of this paper. The formula used for the time-dependent accident consequences was the following:

$$
c_{i j}(t)=w_{1} \cdot\left(\pi \cdot \lambda^{2} \cdot \rho_{i j}\right)+w_{2} \cdot \frac{\left(2 \cdot \lambda \cdot V_{i j}(t)\right)}{l_{i j}}
$$

where $\rho_{i j}$ is the population density along arc $(i, j), \lambda$ is assumed to be equal to 1 mile, $V_{i j}(t)$ is the traffic volume at time $t$ on arc $(i, j), l_{i j}$ is the length of arc $(i, j)$ and $w_{1}$ and $w_{2}$ are the weights for the population density and traffic volume affected by the accident respectively.

\subsubsection{Weights $w_{1}$ and $w_{2}$}

Despite the fact that for each arc we consider the respective population density to be constant throughout the time period understudy, the consideration of the traffic volume at time $t$ gives the desirable dynamic element to the accident consequences. In addition, the weights $w_{1}$ and $w_{2}$ were arbitrary chosen to be 1 and 8 respectively. The reason behind our choice of these values for $w_{1}$ and $w_{2}$ is that by assigning a larger weight on the traffic volume we are enforcing the dynamic element in the $c_{i j}$ 's. As expected, the values of $w_{1}$ and $w_{2}$ affect the results of the model. To see how the model is affected from the values of the weights $w_{1}$ and $w_{2}$ a sensitivity analysis was conducted and is presented in Section 5.3. 


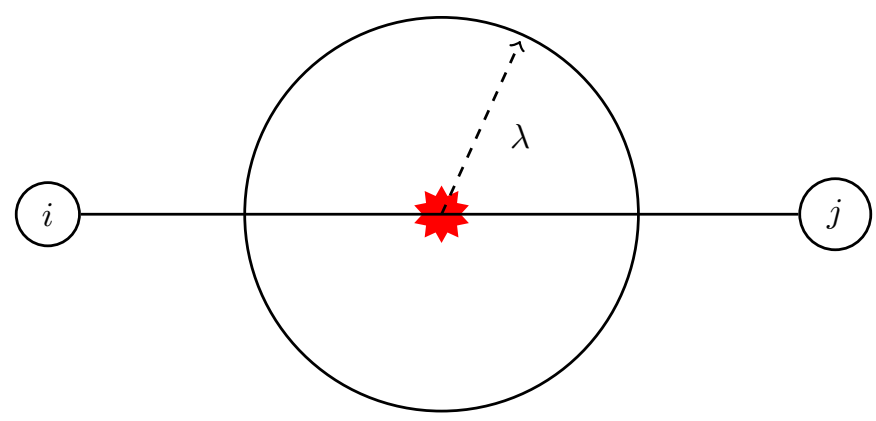

Figure 3: Hazmat accident endangered area described by a circle of radius $\lambda$ in $\operatorname{arc}(i, j)$

\subsection{Numerical Results}

Next we present the results from the case study in the Buffalo network. We will first present the numerical results when the infrastructure of the network was ignored, followed by the results in the case where infrastructure was taken into consideration.

\subsubsection{Accident Consequences Ignoring Infrastructure}

The proposed model was implemented and ran in Matlab R2010a on a 3.10GHz Intel Core i5-2400 CPU computer system. For the particular values of the weights, $w_{1}=1$ and $w_{2}=8$, the maximum computation time required by the algorithm to solve the problem for a single confidence level value was 140 seconds. The model applied in the Buffalo network, results in 10 different optimal routes for the transportation of the hazmat shipment for different $\alpha$ values.

The proposed routes and their respective departure times are shown in Table 3. As shown in this table, the routes maintain their optimality for specific confidence level intervals. Also, note that the model detours more frequently as the confidence level value approaches to one. While the proposed route remains unchanged for confidence level up to 0.9936, beyond that point the optimal path changes repeatedly. This is happening because the accident probabilities are very small, in the range of $10^{-8}$ to $10^{-6}$ (Abkowitz, M. and Cheng, PD, 1988) and the accident consequences of such events are extremely high. Therefore, in order for these events to be captured by the model, it is necessary the confidence level value to approximate 1 .

The graphical representation of the proposed optimal path for each one of the confidence intervals is also provided in Table 3. The first route that the model proposes is for confidence level in the interval $[0,0.9936)$. As already proven in Theorem 1 , when the confidence level $\alpha$ value is close to zero the CVaR model is equivalent to the Traditional Risk model. In other words, the optimal route proposed by the dynamic CVaR model for the particular confidence 
Table 3: Optimal paths given by the CVaR model for various confidence levels $\alpha$

\begin{tabular}{|c|c|c|c|c|c|}
\hline $\begin{array}{l}\text { Confidence } \\
\text { Level } \alpha\end{array}$ & $\begin{array}{c}\text { Departure } \\
\text { Time }\end{array}$ & $\begin{array}{l}\text { Optimal } \\
\text { Route }\end{array}$ & $\begin{array}{l}\text { Confidence } \\
\text { Level } \alpha\end{array}$ & $\begin{array}{c}\text { Departure } \\
\text { Time }\end{array}$ & $\begin{array}{c}\text { Optimal } \\
\text { Route }\end{array}$ \\
\hline$[0,0.9936)$ & 8:55 am & & {$[0.9976,0.9980)$} & 8:50 am & \\
\hline$[0.9936,0.9964)$ & $8: 55$ am & & {$[0.9980,0.9982)$} & $8: 45$ am & \\
\hline$[0.9964,0.9971)$ & $8: 55 \mathrm{am}$ & & {$[0.9982,0.9990)$} & $8: 35$ am & \\
\hline$[0.9971,0.9972)$ & 8:50 am & & {$[0.9990,0.9995)$} & 8:45 am & \\
\hline$[0.9972,0.9976)$ & $8: 50 \mathrm{am}$ & & {$[0.9995,1)$} & 8:40 am & \\
\hline
\end{tabular}


level interval is the same as the one proposed by the dynamic TR model.

Since the dynamic TR model computes the expected value of the risk along a path and manipulates it as a risk measure, the time-dependent CVaR model in this case has a risk-neutral behavior. If we examine the proposed route itself in detail we will find out that it passes from links with high congestion and with moderate population density. The reason for this is that the model fails to capture the extreme events from the risk distribution since the CVaR measure is located at the mean of the distribution. Note that, because the accident probabilities are very small, the upper bound of the interval, 0.9936, is considered very small in hazmat transportation.

At this point we want to emphasize that the final route is the same as the one proposed by the Maximum Risk (MM) model. The final route is proposed when the confidence level is in the interval $[0.9995,1)$ and the optimal CVaR value remains unchanged for all $\alpha$-values in this interval. This is because the MM model minimizes the maximum accident consequences. In other words, because $r=\max \left\{c_{i j}(t):(i, j) \in \mathcal{A}, t \in S\right\}$ from (3) it is clear that the quantity

$$
d_{i j}(t, r)=\sum_{(i, j) \in \mathcal{A}} p_{i j}(t)\left[c_{i j}(t)-r\right]^{+}=0
$$

for all confidence levels $\alpha$. The model in this case has a risk-averse attitude.

This manuscript extends the application of $\mathrm{CVaR}$ as a risk measure in dynamic hazmat routing problems. Comparing the results obtained from the dynamic CVaR model to the respective ones of the static case presented in Toumazis et al. (2013), we can see that the routes proposed in the static case avoid passing through high populated areas. On the other hand, as shown in Figure 2, in the dynamic case the proposed routes avoid the high congested links of the network. Naturally, the level of this behavior depends on the values of the weights $w_{1}$ and $w_{2}$, as demonstrated in Section 5.3.

\subsubsection{Accident Consequences Considering Infrastructure}

Accident consequences can be computed considering many factors. For example in Section 5.2.1, accident consequences were computed considering only the population density around the arcs and the traffic volume on the arcs at the entrance time of the shipment. However, we can calculate the $c_{i j}$ 's by taking into consideration other factors like land value, infrastructures or combination of these factors. For the remainder of this section, the numerical results of a case study considering a combination of population densities, traffic volume and infrastructures for the computation of the accident consequences are presented. The only infrastructures taken into account, are the two bridges that connect Grand Island with Buffalo and Niagara City, and the Buffalo/Niagara International airport. We also assumed that each main infrastructure 
is equivalent to an area with population density equal to 20,000. For easier comparison, we used the same unit for the computation of the accident consequences. expressing the infrastructure cost in terms of population density (Erkut et al., 2007). Note that the number 20,000 was chosen so that the paths passing through the main infrastructures are penalized. In other words, we are imposing penalty to the paths passing by those specific infrastructures. Also to allow these "penalties" to have a bigger effect on the results, we dropped weights $w_{1}$ and $w_{2}$ from the objective function. That is, population densities and traffic volume at the shipments entrance time have the same weight on the objective function.

The network used was exactly the same as before, with the only difference being the different $c_{i j}$ 's values for arcs $(14,18),(21,27)$, which represent the two bridges connecting Grand Island with Niagara City and Buffalo respectively, and arcs $(72,73),(73,74),(73,86)$, $(74,48),(74,75)$ and $(86,48)$, which are the links surrounding the Buffalo-Niagara International airport. At this point we want to emphasize that for the computation of the accident consequences, we used the same approach as before, considering a circle of radius $\lambda=1$ mile as the hazmat accident impact area and the sum of the population density and traffic volume within the affected area.

When all the above are taken under consideration, the proposed algorithm resulted in 6 different paths, each one having its respective departure time. The results are given in Table 4 with the graphical representation of the proposed paths.

Studying the proposed routes, one can easily notice that the behavior of the model in this case is very similar to the case study described in the previous section. However some differences do exist. The resulted routes now avoid the two bridges, i.e. arcs $(14,18)$ and $(21,27)$, as well as the arcs near the airport. In the paths proposed by the model described earlier, every route was passing through either the bridges and/or near the airport.

\subsection{Sensitivity Analysis}

The findings presented in Section 5.2 were obtained when the weights $w_{1}$ and $w_{2}$ are equal to 1 and 8 respectively. As Equation (23) indicates, the weight parameters influence the accident consequence of each link; therefore, they affect the optimal solution as well. This part of the manuscript presents a sensitivity analysis on the values of the weight parameters $w_{1}$ and $w_{2}$ based on the Buffalo network when infrastructure was taken under consideration.

The sensitivity analysis presented next was conducted as follows. The weight corresponding to the population density of each arc, i.e. $w_{1}$, was kept fixed at 1 . On the other hand, the weight corresponding to the traffic conditions, $w_{2}$, was altered between 0 and 1 with step size of 0.1 , and from 1 to 10 with incremental step size of 1 . That is, we tested the optimal 
Table 4: Optimal paths given by the CVaR model for various confidence levels $\alpha$ considering Infrastructure

\begin{tabular}{|c|c|c|c|c|c|}
\hline $\begin{array}{l}\text { Confidence } \\
\text { Level } \alpha\end{array}$ & $\begin{array}{c}\text { Departure } \\
\text { Time }\end{array}$ & $\begin{array}{l}\text { Optimal } \\
\text { Route }\end{array}$ & $\begin{array}{l}\text { Confidence } \\
\text { Level } \alpha\end{array}$ & $\begin{array}{c}\text { Departure } \\
\text { Time }\end{array}$ & $\begin{array}{c}\text { Optimal } \\
\text { Route }\end{array}$ \\
\hline$[0,0.9932)$ & 9:00 am & & {$[0.9969,0.9990)$} & 8:00 am & \\
\hline$[0.9932,0.9967)$ & $8: 55 \mathrm{am}$ & & {$[0.9990,0.9996)$} & 8:55 am & \\
\hline$[0.9967,0.9969)$ & 8:55 am & & {$[0.9996,1)$} & $8: 15 \mathrm{am}$ & \\
\hline
\end{tabular}

solution of the model when the population density is 10 times more important than the traffic conditions, when it is 10 times less important than the traffic conditions, and everything in between. Each pair of weights was tested for the whole range of confidence levels.

Figure 4 presents the effect that weights $w_{1}$ and $w_{2}$ have on a number of different factors. Specifically, Figure 4a presents the effect on the optimal CVaR measure value. As expected, as the value of $w_{2}$ increases, the value of $c_{i j} \forall(i, j) \in \mathcal{A}$ increases. Therefore, since the cost of each path becomes higher as $w_{2}$ value increases, we conclude that a positive correlation exists between the optimal value of the risk measure and the value of $w_{2}$.

Figure 4b shows the impact on the CPU time. Similarly to the previous figure, we notice that as the value of $w_{2}$ increases, the CPU time increases as well. The explanation for this is based once again on the values of the $c_{i j}$ 's. As accident consequences increase, the model's best incumbent optimal value increases as well. Therefore more elements in the ordered set $\widehat{C}$ are tested. However, note that this effect is not significant.

Figure $4 \mathrm{c}$ demonstrates how the proposed departure time is affected. We observe that when the traffic conditions are at least as important as the population density, i.e. the 


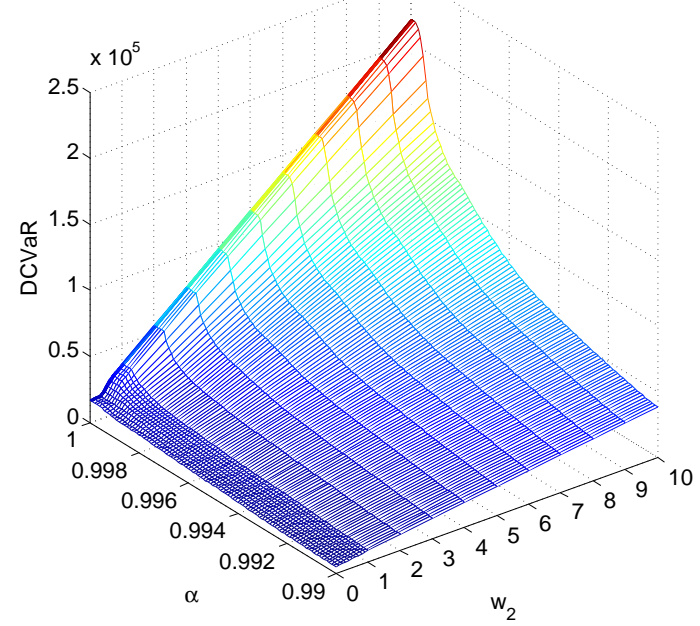

(a) $w_{2}, \alpha, \mathrm{DCVaR}$

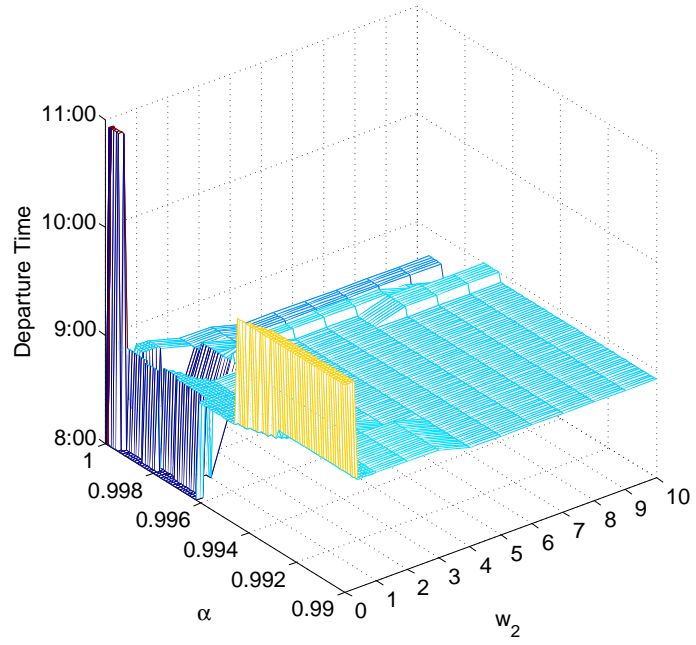

(c) $w_{2}, \alpha$, Departure Time

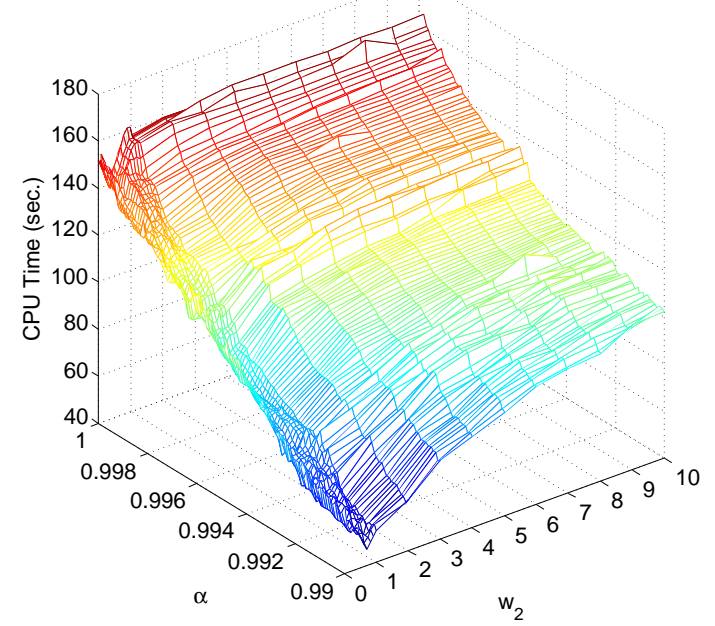

(b) $w_{2}, \alpha$, CPU time

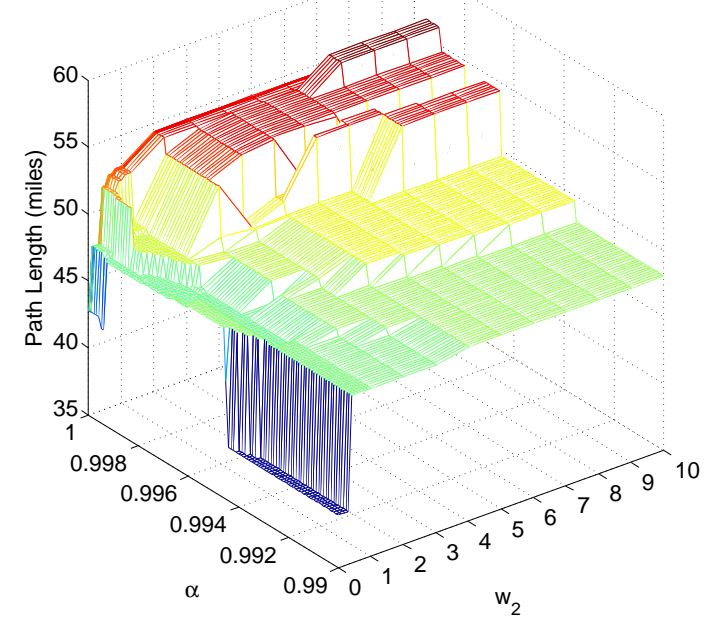

(d) $w_{2}, \alpha$, Path Length

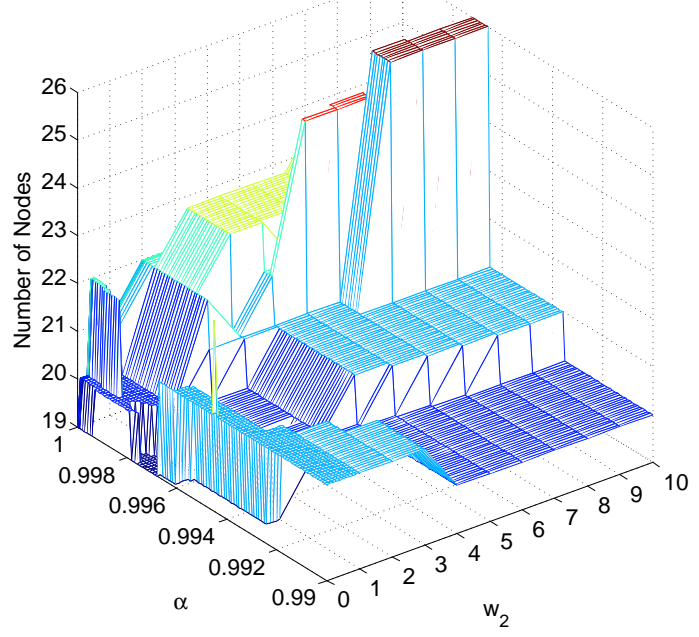

(e) $w_{2}, \alpha$, No. of Nodes

Figure 4: Sensitivity Analysis 
value of $w_{2}$ is large, the departure time exhibits little change. On the other hand, when the traffic conditions are less important than the population density, i.e. value of $w_{2}$ is small, the proposed shipment's departure time fluctuates frequently. In the case where $w_{2}=0$, the problem is the same as the static case, since the population density is the only factor influencing the accident consequences and we assumed the population density is constant. Then, the algorithm ends up with an arbitrary departure time, because all departure times within the entire time horizon bring the same level of risk. Therefore, in such cases the fluctuations in departure time are of no significance. By increasing the weight of the traffic conditions, $w_{2}$, we are enforcing the dynamic element in the model. Since the traffic volume component is added in the computation of the $c_{i j}$ 's, as shown in (23), the value of the accident consequences is increasing as well.

When $w_{2}$ is small, the increase in the value of $w_{2}$ causes frequent changes in the proposed departure time. That is, the model can find a different departure time for the same route that will avoid high traffic congestion. However, when the traffic conditions dominate the population density in the computation of the accident consequences, the proposed departure time stabilizes. This is because the model under these conditions already proposes the best possible departure time. Hence, adding more weight on the traffic conditions will have no impact on the results. In these cases, instead of proposing different departure time, the model avoids the highly congested arcs be following a different path, as presented in Figure 4d.

Finally, Figures $4 \mathrm{~d}$ and $4 \mathrm{e}$ present the effects with respect to the proposed path. From the former figure we can conclude that the value of the parameter $w_{2}$ has a small effect on the proposed path's length, whereas the latter figure shows no correlation between the number of nodes consisting the path and the parameter's value. Furthermore, Figure $4 \mathrm{~d}$ also shows that the model becomes more circuitous as the confidence level value increases; that is, as the model's attitude becomes more risk-averse.

At this point, we revisit Figure $4 \mathrm{~b}$ to compare the theoretical worst-case complexity of the proposed algorithm and the actual running time. As shown in the figure, the proposed method was ran to completion in less than 3 minutes (using Matlab R2010a on a 3.10GHz Intel Core i5-2400 CPU computer system). While the worst-case computational complexity is $O\left(M^{2}|\mathcal{N}|^{3}|\widehat{C}|\right)$, where for the understudy network $\left.M^{2}|\mathcal{N}|^{3}|\widehat{C}|=36^{2} \cdot 90^{3} \cdot[(149 \cdot 36)+1]\right)=$ $5,068,766,160,000$, the actual computation times were less than 3 minutes. The actual CPU time is much less than the theoretical complexity. This may be because the understudy network is far from being a complete network, which is the assumption made in the worst-case computation complexity. In addition, a large portion of $c_{i j}$ values from the set $\widehat{C}$ is skipped by a termination condition of the algorithm. We note that our findings are consistent with the observations of Miller-Hooks and Mahmassani (2000) and Nie and Wu (2009). We emphasize 


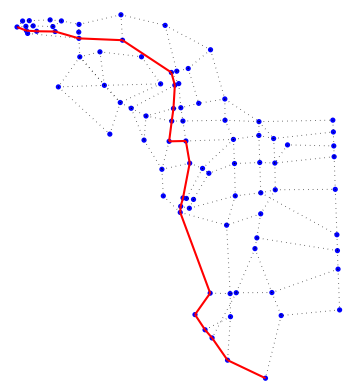

(a) $\alpha=0.9935, w_{1}=1, w_{2}=0.2$

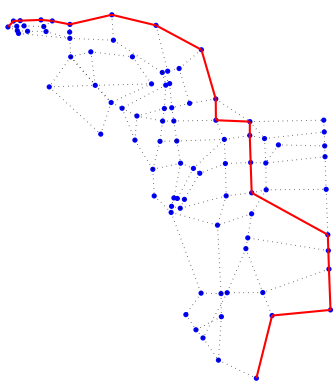

(b) $\alpha=0.9935, w_{1}=1, w_{2}=5$

Figure 5: Optimal Paths at $\alpha=0.9935$ for different $w_{2}$ values

that the proposed algorithm terminates within a practical running time for real networks as well as in a polynomial time in the worst-case.

Figure 5 illustrate the influence of the weights $w_{1}$ and $w_{2}$ on the proposed path. Keeping $\alpha$, and $w_{1}$ fixed, we alter the value of $w_{2}$ from 0.2 to 5 . In other words, firstly the population density was 5 times more important than the traffic conditions and later the population density around the path became 5 times less important than the traffic conditions. As stated previously, in the case where $w_{2}=0$ the model is avoiding the highly populated areas in the network, regardless of the traffic conditions. Hence, a route passing through areas with low population density is acceptable even if there exist highly congested road segments in that particular path. In Figure 5 a we demonstrate the proposed path when $w_{2}=0.2$. At this level, the dynamic element in the model is still week and therefore, high congested arcs are part of the proposed route. As $w_{2}$ is further increased however, these paths are no longer acceptable, because the model's priority is now to avoid the highly congested arcs. Figure 5b illustrates this last-mentioned situation. Comparing the two paths, we can see that initially the model was avoiding the highly populated areas whereas when the change on the weights was made, the model avoids the highly congested links of the network.

\section{Conclusions and Future Work}

This manuscript extends the newly proposed CVaR model applied in hazmat transportation to a static transportation network, to dynamic networks. The objective of this manuscript is to minimize the risk experienced by the hazmat shipment transportation in any given time-dependent transportation network.

We demonstrate the flexibility that the model possesses, which provides the opportunity to the decision makers to retrieve alternative paths for different confidence levels. The decision makers can alter the model's approach from risk-neutral with $\alpha$ close to zero, to risk-averse 
with $\alpha$ close to one. This flexibility of the CVaR model addressing hazmat transportation is something that the existing methods for hazmat transportation are lacking.

Furthermore, CVaR model applied in dynamic networks addresses the problem of risk aversion without adding complexity to the method. Specifically, when compared to the Disutility model (Erkut et al., 2007) that also addresses the problem of risk aversion, CVaR model does not require the difficult task of selecting the risk aversion parameter (Erkut and Ingolfsson, 2000). Also, the nonexistence of that parameter, resolves the numerical problems experienced with the Disutility model that made the population figures extremely big (Erkut and Verter, 1998).

This study suggests that CVaR is a proper risk measure for hazmat route decision making in time-dependent networks. Since the model provides feasible solution relatively fast, it can be utilized for real time hazmat routing decisions. In addition, the CVaR model has the potentials to be used for other known low-probability high-consequence events for risk mitigation.

As previously mentioned, the concept of CVaR applied in hazmat routing has only been recently proposed, and therefore many interesting extensions remain to be further studied. For this research, we focused on a network with a unique origin-destination (OD) pair and a single hazmat shipment. It is in our near future plans, to extend this proposed model to a network with multiple origin-destination pairs and a variety of hazmat shipments with different types of hazmat. That will obviously affect the accident consequences which in that case would also depend on the hazardous material type that is transported.

As previously discussed, for the computation of the accident consequences we used the weighted sum of the affected population along the arcs composing the proposed path and the traffic volume at the time of the accident. However, the values of the weights were arbitrary chosen for this paper. Therefore, an efficient and effective method of determining the weights in a way to represent realistic circumstances is also a potential extension. Furthermore, following the same direction, we can extend the model to the case where the accident consequences capture the population density shifts along each arc. Clearly this will again better address the problem of hazmat routing applied in a more realistic scenario.

In addition, in this paper we assumed that the probability of an accident is the same along each arc, including the intersections, i.e. the nodes. The probability of an accident occurring in an intersection like traffic lights or stop signs is much greater than the probability of an accident occurring in a straight road like the interstates and highways. This improvement also aims to obtain a better representation in a more realistic case.

Finally, for the computation of the accident probabilities and accident consequences, we assumed that there are known (time-variant) parameters. But in reality, this is not the 
case. Due to the fact that hazmat accidents rarely happen, there are not enough data from which we can derive accurate estimates for these parameters. Hence, the computation of the optimal route for hazmat transportation including data uncertainty remains a complicated and challenging issue that needs to be addressed.

\section{Acknowledgement}

This manuscript is based upon work supported by the U.S. Department of Transportation (US DOT), Research and Innovative Technology Administration through the University Transportation Research Center (UTRC) under Grant Number 49111-07-23. The authors are grateful for the support. Any opinions, findings, and conclusions or recommendations expressed in this manuscript are those of the authors and do not necessarily reflect the views of the US DOT or the UTRC. The authors are also thankful to the two anonymous reviewers for their valuable comments that helped to considerably improve the quality of the manuscript.

\section{References}

Abkowitz, M., M. Lepofsky, and P. Cheng (1992). Selecting criteria for designating hazardous materials highway routes. Transportation Research Record 1333, 30-35.

Abkowitz, M. and Cheng, PD (1988). Developing a risk/cost framework for routing truck movements of hazardous materials. Accident Analysis and Prevention 20(1), 39.

Ahuja, R. K., J. B. Orlin, S. Pallottino, and M. G. Scutellà (2002). Minimum time and minimum cost-path problems in street networks with periodic traffic lights. Transportation Science 36, 326-336.

Ahuja, R. K., J. B. Orlin, S. Pallottino, and M. G. Scutellà (2003). Dynamic shortest paths minimizing travel times and costs. Networks 41(4), 197-205.

Andersen, K. A. and A. J. V. Skriver (2000). A label correcting approach for solving bicriterion shortest path problems. Computers \& Operations Research 27, 507-524.

Androutsopoulos, K. and K. Zografos (2010). Solving the bicriterions routing and scheduling problem for hazardous materials distribution. Trasnsportation Research Part C 18, 713-726.

Artzner, P., F. Delbaen, J. Eber, and D. Heath (1999). Coherent measures of risk. Mathematical Finance 9(3), 203-228. 
Bianco, L., M. Caramia, and S. Giordani (2009). A bi-level flow model for hazmat transportation network design. Trasnsportation Research Part C: Emergency Technologies 17, $175-196$.

Branston, D. (1976). Link capacity functions: A review. Transportation Research 10, 223-236.

Chabini, I. and S. Ganugapati (2002). Parallel algorithms for dynamic shortest path problems. International Transactions in Operational Research 9, 279-302.

Chang, T., L. Nozick, and M. Turnquist (2005). Multiobjective path finding in stochastic dynamic networks, with application to routing hazardous materials shipments. Transportation science $39(3), 383$.

Chen, A. and Z. Zhou (2010). The $\alpha$-reliable mean-excess traffic equilibrium model with stochastic travel times. Transportation Research Part B 44, 493-513.

Chen, G., M. S. Daskin, Z.-J. M. Shen, and S. Uryasev (2006). The $\alpha$-Reliable Mean-Excess Regret Model for Stochastic Facility Location Modeling. Wiley Periodicals, Inc. Naval Research Logistics 53, 617-626.

Cooke, K. and E. Halsey (1966). The shortest route through a network with time-dependent intermodal transit times. Journal of Math Analysis and Applications 17, 492-498.

Dial, R., F. Glover, Karney, and D. Klingman (1979). A Computational Analysis of Alternative Algorithms and Labeling Techniques for Finding Shortest Path Trees. Networks 9, 215-248.

Dowd, K. and D. Blake (2006). After VaR: The Theory, Estimation, and Insurance Applications of Quantile-Based Risk Measures. Journal of Risk and Insurance 73(2), 193-229.

Dreyfus, S. (1969). An Appraisal of Some Shortest-Path Algorithms. Operations Research 17, 395-412.

Einhorn, D. (2008). Private profits and socialized risk. Global Association of Risk Professionals Risk Review June/July.

Erkut, E. and O. Alp (2007). Designing a road network for hazardous materials shipments. Computers $\&$ Operations Research 34, 1389-1405.

Erkut, E. and F. Gzara (2008). Solving the hazmat transport network design problem. Computers 83 Operations Research 35, 2234-2247.

Erkut, E. and A. Ingolfsson (2000, May). Catastrophe avoidance models for hazardous materials route planning. Transportation Science 34(2), 165-179. 
Erkut, E. and A. Ingolfsson (2005, January). Transport risk models for hazardous marterials: revisited. Operations Research Letters 33, 81-89.

Erkut, E., S. Tjandra, and V. Verter (2007). Hazardous materials transportation. In C. Barnhart and G. Laporte (Eds.), Operations Research Handbook on Transportation, pp. 539-611.

Erkut, E. and V. Verter (1998). Modeling of transport risk for hazardous materials. Operations Research, 625-642.

Federal Motor Carrier Safety Administration (2001). Comparative Risks of Hazardous Materials and Non-Hazardous Materials Truck Shipment Accidents/Incidents.

Federal Motor Carrier Safety Administration (2006). Nine Classes of Hazardous Materials.

Harwood, D., J. Viner, and E. Russell (1993). Procedure for developing truck accident and release rates for hazmat routing. Journal of Transportation Engineering 119, 189-199.

Jin, H. and R. Batta (1997). Objectives derived from viewing hazmat shipments as a sequence of independent bernoulli trials. Transportation Science 31(3), 252-261.

Kang, Y., R. Batta, and C. Kwon (2013a). Generalized Route Planning Model for Hazardous Material Transportation with VaR and Equity Considerations. Working Paper.

Kang, Y., R. Batta, and C. Kwon (2013b). Value-at-Risk Model for Hazardous Material Transportation. Annals of Operations Research Accepted.

Kara, B. and V. Verter (2004, May). Designing a road network for hazardous materials transportation. Transportation Science 38(2), 188-196.

Kwon, C., T. Lee, and P. Berglund (2013). Robust shortest path problems with two uncertain multiplicative cost coefficients. Naval Research Logistics 60(5), 375-394.

Lo, H., X. Luo, and B. Siu (2006). Degradable transport network: travel time budget of travelers with heterogeneous risk aversion. Transportation Research Part B 40, 792-806.

Miller-Hooks, E. and H. Mahmassani (1998). Optimal routing of hazardous materials in stochastic, time-varying transportation networks. Transportation Research Record: Journal of the Transportation Research Board 1645(-1), 143-151.

Miller-Hooks, E. D. and H. S. Mahmassani (2000, May). Least Expected Time Paths in Stochastic, Time-Varying Transportation Networks. Transportation Science 34(2), 198-215. 
Nie, Y. and X. Wu (2009). Shortest path problem considering on-time arrival probability. Transportation Research Part B 43, 597-613.

Nocera, J. (2009). Risk mismanagement. The New York Times Magazine January 4, 2009.

Pflug, G. (2000). Probabilistic Constrained Optimization: Methodology and Applications. Kluwer Academic Publishers.

ReVelle, C., J. Cohon, and D. Shobrys (1991). Simultaneous siting and routing in the disposal of hazardous wastes. Transportation Science 25(2), 138.

Rockafellar, R. and S. Uryasev (2002). Conditional value-at-risk for general loss distributions. Journal of Banking \& Finance 26(7), 1443-1471.

Rockafellar, R. T. and S. Uryasev (2000). Optimization of Conditional Value-at-Risk. Journal of Risk 2(3), 21-42.

Saccomanno, F. and A. Chan (1985). Economic evaluation of routing strategies for hazardous road shipments. Transportation Research Record 1020, 12-18.

Sarykalin, S., G. Serraino, and S. Uryasev (2008). Value-at-risk vs. conditional value-at-risk in risk management and optimization. Tutorials in Optimization Research, 270-294.

Shao, H., W. Lam, Q. Meng, and M. L. Tam (2006). Demand driven travel time reliabilitybased traffic assignment problem. Transportation Research Record 1985, 220-230.

Sherali, H., L. Brizendine, T. Glickman, and S. Subramanian (1997). Low probabilityhigh consequence considerations in routing hazardous material shipments. Transportation Science $31(3), 237-251$.

Sivakumar, R. A., B. Rajan, and M. Karwan (1993). A network-based model for transporting extremely hazardous materials. Operations Research Letters 13(2), 85-93.

Toumazis, I. and C. Kwon (2013). Worst-case conditional value-at-risk minimization for hazardous materials transportation. Working Paper.

Toumazis, I., C. Kwon, and R. Batta (2013). Value-at-risk and conditional value-at-risk minimization for hazardous materials routing. In R. Batta and C. Kwon (Eds.), Handbook of $\mathrm{OR} / \mathrm{MS}$ Models in Hazardous Materials Transportation. Springer.

Transportation Research Board (2005). Special Report 283: Cooperative Research for Hazardous Materials Transportation: Defining the Need, Converging on Solutions. Washington, D.C.: National Research Council. 
U.S. Census Bureau (2007a). 2007 Commodity Flow Survey - Hazardous Materials Report. Technical report, U.S. Department of Transportation and U.S. Department of Commerce.

U.S. Census Bureau (2007b). 2007 Commodity Flow Survey - Summary Report. Technical report, U.S. Department of Transportation and U.S. Department of Commerce.

U.S. Department of Transportation (1998). Hazardous materials shipments. Technical report, Research and Special Programs Administration Office of Hazardous Materials Safety.

Verter, V. and B. Y. Kara (2008). A path-based approach for hazmat transport network design. Management Science 54, 29-40.

Wu, X. and Y. M. Nie (2011). Modeling heterogenous risk-taking behavior in route choice: A stochastic dominance approach. Procedia Social and Behavioral Sciences 17, 382-404.

Xie, Y., W. Lu, W. Wang, and L. Quadrifoglio (2012). A multimodal location and routing model for hazardous materials transportation. Transportation Research Part C 227-228, $135-141$.

Zhou, Z. and A. Chen (2008). Comparative analysis of three user equilibrium models under stochastic demand. Journal of Advanced Transportation 42, 239-263.

Ziliaskopoulos, A. K. and H. S. Mahmassani (1993). Time-Dependent, Shortest-Path Algorithm for Real-Time Intelligent Vehicle Highway System Applications. In Transportation Research Record: Journal of the Transportation Research Board, No. 1408, pp. 94-100. Transportation Research Board of the National Academies, Washington, D.C.

Zografos, K. and K. Androutsopoulos (2008). A decision support system for integrated hazardous materials routing and emergency response decisions. Transportation Research Part C: Emerging Technologies 16(6), 684-703.

Zografos, K., K. N. Androutsopoulos, and V. G. M. (2002). A real-time decision support system for roadway network incident response logistics. Transportation Research Part C 10, 1-18. 


\section{A Proofs}

\section{A.1 Proof of Lemma 1}

Proof. Consider a path $l \in \mathcal{P}$ with $\mathrm{VaR}_{\alpha}^{l}=0$. Then we have,

$$
\lambda_{\alpha}^{l}=\operatorname{Pr}\left[R^{l} \leq \operatorname{VaR}_{\alpha}^{l}\right]=\operatorname{Pr}\left[R^{l} \leq 0\right]=1-\sum_{(i, j) \in \mathcal{A}^{l}} p_{i j}
$$

Therefore, we obtain

$$
\mathrm{CVaR}_{\alpha}^{l}=\lambda_{\alpha}^{l} \operatorname{VaR}_{\alpha}^{l}+\left(1-\lambda_{\alpha}^{l}\right) \mathbb{E}\left[R^{l} \mid R^{l}>\operatorname{VaR}_{\alpha}^{l}\right]=\left(\sum_{(i, j) \in \mathcal{A}^{l}} p_{i j}\right) \mathbb{E}\left[R^{l} \mid R^{l}>0\right]
$$

Using conditional probabilities, we can write:

$$
\begin{aligned}
\mathbb{E}\left[R^{l}\right] & =\mathbb{E}\left[R^{l} \mid R^{l}>\mathrm{VaR}_{\alpha}^{l}\right] P\left(R^{l}>\mathrm{VaR}_{\alpha}\right)+\mathbb{E}\left[R^{l} \mid R^{l}=\mathrm{VaR}_{\alpha}\right] P\left(R^{l}=\mathrm{VaR}_{\alpha}\right) \\
& =\mathbb{E}\left[R^{l} \mid R^{l}>0^{l}\right] P\left(R^{l}>0\right)+\mathbb{E}\left[R^{l} \mid R^{l}=0\right] P\left(R^{l}=0\right)
\end{aligned}
$$

Since $\mathbb{E}\left[R^{l} \mid R^{l}=0\right]=0$, we obtain

$$
\mathbb{E}\left[R^{l} \mid R^{l}>0\right]=\frac{\mathbb{E}\left[R^{l}\right]}{P\left(R^{l}>0\right)}=\frac{\sum_{(i, j) \in \mathcal{A}^{l}} p_{i j} c_{i j}}{\sum_{(i, j) \in \mathcal{A}^{l}} p_{i j}}
$$

Hence, $\mathrm{CVaR}_{\alpha}^{l}$ is

$$
\begin{aligned}
\mathrm{CVaR}_{\alpha}^{l} & =\left(\sum_{(i, j) \in \mathcal{A}^{l}} p_{i j}\right) \frac{\sum_{(i, j) \in \mathcal{A}^{l}} p_{i j} c_{i j}}{\sum_{(i, j) \in \mathcal{A}^{l}} p_{i j}} \\
& =\sum_{(i, j) \in \mathcal{A}^{l}} p_{i j} c_{i j}
\end{aligned}
$$

which completes the proof.

\section{A.2 Proof of Lemma 2}

Proof. Consider a path $l \in \mathcal{P}$ with $\operatorname{VaR}_{\alpha}^{l}=\max _{(i, j) \in \mathcal{A}^{l}} c_{i j}$. Then we have,

$$
\lambda_{\alpha}^{l}=\operatorname{Pr}\left[R^{l} \leq \operatorname{VaR}_{\alpha}^{l}\right]=\operatorname{Pr}\left[R^{l} \leq \max _{(i, j) \in \mathcal{A}^{l}} c_{i j}\right]=1
$$


Therefore, we obtain

$$
\mathrm{CVaR}_{\alpha}^{l}=\lambda_{\alpha}^{l} \operatorname{VaR}_{\alpha}^{l}+\left(1-\lambda_{\alpha}^{l}\right) \mathbb{E}\left[R^{l} \mid R^{l}>\operatorname{VaR}_{\alpha}^{l}\right]=\operatorname{VaR}_{\alpha}^{l}=\max _{(i, j) \in \mathcal{A}^{l}} c_{i j}
$$

Hence proof.

\section{A.3 Proof of Theorem 1}

Proof. Let us define

$$
\alpha^{l}=1-\sum_{(i, j) \in \mathcal{A}^{l}} p_{i j} \quad \text { and } \quad \alpha_{\min }=\min _{l \in \mathcal{P}} \alpha^{l}
$$

Then, $\operatorname{VaR}_{\alpha}^{l}=0$ for all $\alpha \in\left(0, \alpha^{l}\right.$ ] (Kang et al., 2013a) and for each $l \in \mathcal{P}$. Therefore, $\operatorname{VaR}_{\alpha}^{l}=0$ for all $\alpha \in\left(0, \alpha_{\text {min }}\right]$ and for all path $l \in \mathcal{P}$. Then, by Lemma 1 ,

$$
\mathrm{CVaR}_{\alpha}^{l}=\sum_{(i, j) \in \mathcal{A}^{l}} p_{i j} c_{i j}
$$

for all $\alpha \in\left(0, \alpha_{\min }\right]$ and for all path $l \in \mathcal{P}$. Therefore minimizing the CVaR measure is identical to minimizing the Traditional Risk measure.

\section{A.4 Proof of Theorem 2}

Proof. Kang et al. (2013a) showed that for each path $l \in \mathcal{P}, \operatorname{VaR}_{\alpha}^{l}=\max _{(i, j) \in \mathcal{A}^{l}} c_{i j}$ for all $\alpha \in\left(p_{\max }^{l}, 1\right)$. Therefore, by Lemma 2 , we have $\mathrm{CVaR}_{\alpha}^{l}=\max _{(i, j) \in \mathcal{A}^{l}} c_{i j}$ for all $\alpha \in\left(p_{\max }^{l}, 1\right)$ and for each path $l \in \mathcal{P}$.

Consequently,

$$
\mathrm{CVaR}_{\alpha}^{l}=\max _{(i, j) \in \mathcal{A}^{l}} c_{i j}
$$

for all $\alpha \in\left(\alpha_{\max }, 1\right)$ and for all path $l \in \mathcal{P}$ where $\alpha_{\max }=\max _{l \in \mathcal{P}} p_{\max }^{l}$. Therefore, the CVaR minimization is equivalent to the Maximum Risk minimization.

\section{A.5 Proof of Lemma 3}

Proof. Let us consider any binary $x^{\prime}$, and the following problem:

$$
\min _{r \in \mathbb{R}^{+}}\left(r+\frac{1}{1-\alpha} \sum_{(i, j) \in \mathcal{A}^{l}} d_{i j}\left(\theta_{i j}^{l}(t), r\right) x_{i j}^{\prime}\right)
$$


From the definition of $d_{i j}\left(\theta_{i j}^{l}(t), r\right)$ we know that

$$
d_{i j}\left(\theta_{i j}^{l}(t), r\right)=\sum_{(i, j) \in \mathcal{A}} p_{i j}\left(\theta_{i j}^{l}(t)\right)\left[c_{i j}\left(\theta_{i j}^{l}(t)\right)-r\right]^{+}
$$

Hence we can write the above problem in the following form:

$$
\min _{r \in \mathbb{R}^{+}}\left(r+\frac{1}{1-\alpha} \sum_{(i, j) \in \mathcal{A}^{l}} p_{i j}\left(\theta_{i j}^{l}(t)\right)\left[c_{i j}\left(\theta_{i j}^{l}(t)\right)-r\right]^{+} x_{i j}^{\prime}\right)
$$

Let $\widehat{C}$ be an ordered set of $\{0\} \cup\left\{c_{i j}\left(\theta_{i j}^{l}(t)\right):(i, j) \in \mathcal{A}, t \in S\right\}$ and $\widehat{c}_{k}$ denote $k$-th smallest element of $\widehat{C}$, where $\widehat{c}_{0}=0$. Then the above minimization problem is equivalent to the following problem:

$$
\min _{k \in\{0,1, \ldots,|\mathcal{A}| \times|S|-1\}} \min _{r_{k} \in\left[\widehat{c}_{k}, \widehat{c}_{k+1}\right]}\left(r_{k}+\frac{1}{1-\alpha} \sum_{(i, j) \in \mathcal{A}_{1}\left(r_{k}\right)} p_{i j}\left(\theta_{i j}^{l}(t)\right)\left[c_{i j}\left(\theta_{i j}^{l}(t)\right)-r_{k}\right] x_{i j}^{\prime}\right)
$$

where $\mathcal{A}_{1}\left(r_{k}\right)=\left\{(i, j): c_{i j}\left(\theta_{i j}^{l}(t)\right)>r_{k}\right\}$. Note that we eliminated + operators. For each $k$, the inner problem of $r$ is a linear optimization problem with a simple closed interval constraint. Therefore, $r_{k}^{*}$ is either $\widehat{c}_{k}$ or $\widehat{c}_{k+1}$. Since $x^{*}$ to the CVaR minimization problem should be binary and the choice of $x^{\prime}$ is arbitrary, we obtain the lemma. 